\title{
Theoretical aspects of aerospace image processing in quasi two- dimensional spectral space
}

\author{
B.V. Kostrov ${ }^{1}$, D.V. Grigorenko ${ }^{1}$, V.N. Ruchkin ${ }^{2}$ and V.A. Fulin ${ }^{2}$ \\ ${ }^{1}$ Ryazan State Radio Engineering University, Ryazan, Russian Federation \\ ${ }^{2}$ Ryazan State University, Ryazan, Russian Federation
}

\begin{abstract}
The article regards digital images as mathematical objects and uses a co-variation matrix of element brightness values to analyze an autocorrelation matrix of images - a dispersion matrix, to define all significant properties of images with the help of mathematical statistics and matrix computing. We suggest theoretic and methodological generalizations in the field of aerospace photo processing, basing on choice of the most advantageous variant of constructing basic functions among various Vilenkin-Chrestenson functions (VCF) in a non-trigonometrical minimally available construction form as Walsh functions with an original 'carcass' of Rademacher functions. The methodology of this research is based on a set of theorems that are different from corresponding theorems of classical spectral analysis [5]. The conclusions of the theorems allow constructing effective algorithms of filtration and correlation analysis of aerospace images when noises and distortions appear.
\end{abstract}

\section{Introduction}

An image can be regarded as a discrete signal, defined at a finite interval of its coordinates in a plane. The best results in aerospace image processing are now obtained via algorithms in spatial coordinates, where space and frequency properties of images are ignored. Information about using classical harmonic analysis in image processing is available in [1]. However, the complexity of implementation in Fournier transforms prevents these methods from becoming popular tools in aerospace image processing. For solving tasks of spectral analysis in general cases we can use any systems that contain a necessary number of orthogonal functions. Choosing the system functions will be based on convenient computing and ultimately optimize labour input necessary for implementation of a required transform. The application of alternative basic function systems requires a methodological analysis of their use for solving such tasks.

The paper generalizes the theory and methodology of previous research by both authors, which allows expanding the methodology of using orthogonal transformations in the field of aerospace image processing. In this way, a theoretical and methodological basis is made for using systems of Vilenkin-Chrestenson functions (VCF) in a non-trigonometrical minimally available construction form as Walsh functions, with an original 'carcass' of Rademacher functions. The resulting theoretical and methodological statements are necessary for development of algorithms of filtration and correlational analysis of aerospace images when noises and distortions appear.

The paper is organized as follows. Section 2 treats an aerospace image as a mathematical object, a co-variation matrix of brightness values, and autocorrelation matrix of images - dispersion matrix - all significant properties of images are described with the help of mathematical statistics and matrix computing. Section 3 defines the main idea and theoretical pre-requisites for solving the tasks with the help of Vilenkin-Chrestenson functions (VCFs) in a non-trigonometrical minimally available construction form as Walsh functions, with an original 'carcass' of Rademacher functions. Section 4 presents a methodology of using such transforms, it is based on a set of theorems that are different from corresponding theorems of classical spectral analysis [5]. In Section 5, the authors describe some experimental results of using theorems 1 and 5 for filtration of aerospace images with synchronous multiplicative noises and provide recommendations for software and hardware implementation of spectral representation of aerospace images in programmable logic integral circuits (PLICs).

\section{Principal results}

As far as the principles and tools of aerospace image photography (ASI) [2] are concerned, all video sensors in radio systems can be divided into 5 groups:

Group 1 includes sensors that are constructed on the basis of charge coupled devices (CCDs);

Group 2 includes single-beam sensors with conical or flat-bed scanning; 
Group 3 includes scanning sensors;

Group 4 consists of radio-locator stations with various bases and operational principles;

Group 5 can include thermal imagery devices and televisions, as well as systems that are typically mounted on flying apparatuses and on on-ground stations.

All the listed methods and systems create panchromatic video data that can be fixed or saved in a number of ways. They all have a certain geometrical shape, and their content is represented as densities of blacker areas in video; these data can be transferred via various systems, and they all share properties that allow referring to them as aerospace images (ASIs).

To solve tasks in analysis and processing of ASIs, it is necessary to define 'image' as a mathematical object possessing certain analytical properties. Currently, performing mathematical operations on an image presupposes that it exists in digital form, as a digital image [2], represented as a discrete array of numbers, forming a matrix of elements, $b_{i j}$ with parameters $(i, j)$, where $1 \leq i \leq n$ and $1 \leq j \leq n$. Elements $b_{i j}$ are squares (apertures, pixels), defined by inequalities $i-1 \leq x<i$ and $j-1 \leq y<i \quad(x$ and $y$ are spatial co-ordinates of a continuous image). Then any $\left[b_{i j}\right]_{\text {matrix of a } n \times n}$ can be assigned an image, whose brightness value squared $[i-1 \leq x<i] \times[j-1 \leq y<i]$ equals a constant value $\bar{b} i j$ for each pair $(i, j)$. A digital image element can be represented as a random value $\widetilde{b}_{i j}$, and the $\left[\widetilde{b}_{i j}\right]$ matrix of random values. As the $b_{i j}$ values can be taken as full representatives of random values $\widetilde{b}_{i j}$, we transpose their value onto the area of the whole element.

The sum of random values of the image element brightnesses leads to a value of $\Sigma_{B}=M\left\{(B-\bar{B})(B-\bar{B})^{\prime}\right\}$, where $M$ is an operator of mathematical expectation, $\bar{B}$ corresponds to the vector average, ${ }^{(B-\bar{B})}$ represents the column matrix, and $(B-\bar{B})^{\prime}$ stands for a row matrix.

The obtained matrix contains $n^{2} \times n^{2}$ elements, the diagonal elements are dispersions of specific random values, and all the other elements correspond to covariations of two random values $b_{i j}$, measured at different values of $i$ and $j$. A co-variation matrix is completely symmetrical.

The utility of an image can be defined via analysis of a self-correlation image matrix, also called a dispersion matrix, as is characterizes the image element dispersion.

$$
S_{B}=\left[\begin{array}{ccc}
M\left\{b_{11}^{2}\right\} & \ldots & M\left\{b_{11} b_{n n}\right\} \\
\ldots & \ldots & \ldots \\
M\left\{b_{n n} b_{11}\right\} & \ldots & M\left\{b_{n n}^{2}\right\}
\end{array}\right]
$$

In some cases, the $\Sigma_{B}$ matrix is more conveniently represented via correlation coefficients, defined as $r_{i j}=\sigma_{i j}^{2} / \sigma_{i i} \sigma_{j j}$. Then $\Sigma_{B}$ will equal $\Sigma_{B}=\Gamma_{\eta} R_{K} \Gamma_{\eta}$, where
$\Gamma_{\eta}$ is a diagonal matrix of mean square deviations $\sigma_{i j}$, and $R_{K}$ is a matrix of correlation coefficients $r_{i j}$.

$$
\Sigma_{B}=\sigma^{2} R_{K}^{\prime}, \mathrm{R}_{\mathrm{K}}^{\prime}=\left[\begin{array}{cccc}
1 & r_{1} & \ldots & r_{n-1} \\
r_{1} & \ldots & \ldots & \ldots \\
\ldots & \ldots & \ldots & \ldots \\
r_{n-1} & \ldots & \ldots & \ldots
\end{array}\right]_{(2)},
$$

where is a correlation matrix for rows and columns,

and $r_{i}$ are correlation coefficients for rows and columns.

Thus, using tools and methods of mathematical statistics and matrix computing, we can define all significant properties of images.

ASIs develop specific distortions due to their formation principles and conditions of transfer, and these distortions can be classified:

- synchronous distortions due to changing transfer properties of the pixel brightness formation tract: these are rigidly connected to the law of scanning ASIs (specific 'synchronous stripes' along the rows or columns);

- distortions as group noises that can behave absolutely destructively (shown as missing pixels along ASI rows);

- non-synchronous distortions not related to the pixel brightness formation tract of the scanning law (shown as specific periodic 'non-synchronous stripes' located at a random angle to the ASI columns).

Removal of described distortions is the primary task of preliminary processing of compression algorithm picture compression, to form prerequisites for using the images).

\section{Main idea and theoretical pre- requisites for solving the tasks}

The existence of an image digital form defines mathematical tools available for execution of processing operations. These are mostly discrete transformations defined for finite intervals. Introducing the notion of finiteness, we can avoid the contradictions that arise from the Fourier transformation, and conduct spatial-spectral analysis of images that are in a general way described by a non-stationary arbitrary process. In this respect, using the principal statements and conclusions of the theory of discrete signal at finite intervals is quite justified. This theory is based on choosing the best acceptable option of constructing a basic function system from the whole range of VCFs, for which signal shift is defined as bitwise addition of numbers in a certain module. The notion of VCF includes Walsh function systems (based on binary arithmetic) as an individual case.

The methodology of applying this system at an interval is based on the following properties:

1. All functions of the system are valid for definition interval $_{N}=2^{n}$.

2. As the system function values are limited to +1 and -1 , the basic operations for expansion in a Walsh system are addition and subtraction. 
3. The Walsh function remains orthogonal at the definition interval of $N$, and the Hadamard matrix built according to Walsh functions is symmetrical.

4. As the Hadamard matrix is sized $N \times N$, it includes $N$ orthogonal functions and therefore no new orthogonal functions can be added. This means that such a function system is complete and can be used for unitary transformations of non-harmonic spectral analysis. The computational complexity of such transformations will be minimal, as all the operations will be replaced with addition of real numbers, and not of discrete exponential functions, where all numbers are complex [3].

Alternatively, VCFs can also be represented by Rademacher functions (complex) for the same interval $N=m^{n}$ :

$$
R_{i}(x)=e^{j(2 \pi / m) x_{i}}
$$

where the $i$ index stands for the order of a Rademacher function.

Then, in accordance with (1), VCFs can be written as

$$
F(p, x)=\prod_{i=0}^{n-1}\left[R_{i}(x)\right]<P_{i}>
$$

For the case when $m=2$, the Rademacher function can be set like this:

$$
r_{i}(x)=(-1)^{<x_{i}>}
$$

where $\left\langle x_{i}\right\rangle$ is the $i$ order of a binary representation of the $x$ variable.

In such a task, all Rademacher functions are valid and uneven functions for the $N$ interval. The system that they constitute in not complete. Its completion results in a Walsh function system:

$$
\operatorname{wal}(w, x)=\prod_{i=1}^{n}\left[r_{i}(x)^{<w_{i}>}\right]
$$

where $\left\langle w_{i}\right\rangle$ is the value of the $i$ order of a Rademacher function number, represented in a Gray code: $i=1,2,3, \ldots, n$.

If the number of the Rademacher function contains only one unit, the Walsh function will coincide with the Rademacher function having the same number.

Thus, for definition interval $N=2^{n}$ a Walsh function system can be divided into $n$ groups (here, the zero order function is not considered). If these groups are numbered $k=1,2,3, \ldots, n$, each group begins with Rademacher function $r_{n+1-k}$, and each includes $2^{n-k}$ functions (including the Rademacher function proper). Thus, a Rademacher function system forms a 'carcass' on which the Walsh system is built; and this circumstance has to be considered for spectral analysis of signals.

The methodology of this research is based on a set of theorems that are different from corresponding theorems of classical spectral analysis [5]. The conclusions of the theorems allow constructing effective algorithms of filtration and correlation analysis of aerospace images when noises and distortions appear.

\section{Methodology of aerospace image processing}

2. Theorem on dyad convolution

If $\left\{X_{n}\right\}$ and $\left\{Y_{n}\right\}$ are digital sequences set for an $N$ interval, the sequence of convolution (correlation) $\left\{Z_{s}\right\}$ :

will be as follows:

$$
Z_{s}=\frac{1}{N} \sum_{n=0}^{N-1} X_{n} Y_{n \oplus S}
$$

$$
Z_{s}=\sum_{u=0}^{N-1} N\left(C_{u}^{X} \times C_{u}^{Y}\right)
$$

where $C_{u}^{X}$ are spectral coefficients of the $\left\{X_{n}\right\}$ sequence, and $C_{u}^{Y}$ are spectral coefficients of the $\left\{Y_{n}\right\}$ sequence.

The theorem gives an insight into the mechanism of formation of signal elements following their filtration in a spectral space.

2. Theorem on realnumber-dyad convolution (RDC)

If $\left\{X_{n}\right\}$ and $\left\{Y_{n}\right\}$ are digital sequences set for an $\mathrm{N}$

interval, the sequence of convolution (correlation) $\left\{Z_{s}\right\}$

$$
Z_{S}=\frac{1}{N} \sum_{n=0}^{N-1} X_{n} Y_{n-s}
$$

can be computed as follows:

$$
Z_{s}=\sum_{u=0}^{N-1} C_{u}^{X}\left(C_{u}^{Y}\right)_{s}
$$

where $C_{u}^{X}$ are spectral coefficients of the $\left\{X_{n}\right\}$ sequence, and $\left(C_{u}^{Y}\right)_{s}$ are spectral coefficients computed after a sequence shift $\left\{Y_{n}\right\}$.

This theorem allows constructing complex spectral filters and effective algorithms for correlation analysis. The proof of the theorem and methods of construction of a correlation function for two images can be found in [4]. Physical foundations of RDF functioning are given in $[5$, 6].

\section{Theorem on invariance of thinned basis}

If $\left\{V_{n}\right\}$ is a digital sequence obtained from sequence $\left\{X_{n}\right\}$ through a dyad shift by value of $l$, i.e. $V_{n}=X_{n \oplus l}$,

$$
\text { and } C^{v}=\frac{1}{N} \mathbf{H}_{\mathbf{w}} \mathbf{V} \text {, and } C^{x}=\frac{1}{N} \mathbf{H}_{\mathbf{w}}^{\mathbf{0}} \mathbf{X},
$$

where $C^{v}$ are coefficients of the Walsh sequence spectrum, $\left\{V_{n}\right\} C^{x}$ are coefficients of the sequent spectrum of the $\left\{X_{n}\right\}$ sequence, $\mathrm{H}_{\mathrm{w}}^{0}$ is an arbitrarily thinned Hadamard matrix, and $\mathbf{H}_{\mathbf{w}}$ is the initial matrix, i.e. $\left(C^{v}\right)^{2}=\left(C^{x}\right)^{2}$.

This theorem allows certain filtration types while obtaining spectral presentations, which is not typical of other basic function systems. Proof of this theorem is given in [7].

4. Theorem on restriction of a non-trigonometrical spectrum

If $n$ is the order of a Rademacher function system, and $k$ is the number of a function groupd in a Walsh system, restriction of the spectrum is applied at the level Rademacher functions, number of $n+1-k$, then in the newly formed image the element brightness $b_{i j}^{\left[N_{s}\right]}$ will be: 


$$
b_{i j}^{\left[N_{s}\right]}=\frac{1}{S^{2}} \sum_{g=1}^{s} \sum_{p=1}^{s} b_{g p},
$$

where $N_{S}=\frac{N}{S}, s_{s=2^{k-1}}$, and $i, j=\overline{1, N_{S}}$. Here, the $i(s) ; j(s) ; g(i) ; p(j)$ dependencies are ignored for simplification of the formula.

Intuitive use of this property allows constructing a two-level algorithm of image alignment, requiring computational costs that are tenfold lower as compared to algorithms described in [5]. An implementation of this approach is discussed in $[8,15]$.

5. Theorem on energetic completeness of a quasi-twodimensional spectral presentation

If $b$ is the matrix of a digital image sized $M \times N$, and $C$ are coefficients of its quasi-two-dimensional spectral representation, this equality is true:

$$
\frac{1}{M N} \sum_{i=0}^{M-1} \sum_{j=0}^{N-1} b_{i j}^{2}=\frac{1}{M} \sum_{i=0}^{M-1} \sum_{v=0}^{N-1} C_{i v}^{2}
$$

Expression (2) is a Parseval equation for a quasi-twodimensional presentation of two-dimensional signals. Its proof does not require any commentary, as it results from certain properties of spectral analysis. Using this theorem allows twofold reduction of computing effort and several other benefits, through removal of certain distortion types [9].

\section{Theorem on a constant component of spectrum}

If we assume that $b(x, 0)=0$ is a concrete value of an image element in every row, it is not necessary to transfer the spectral constant component via a communications channel, because it can always be recovered with this formula:

$$
B_{0}=-\sum_{v=1}^{N-1} B(x, v-1)(-1) \sum_{i=1}^{n}\left\langle(v-1)_{i} x_{i}\right\rangle
$$

In this case, the transferred spectrum does not have a constant component.

\section{Experimental results of image filtration}

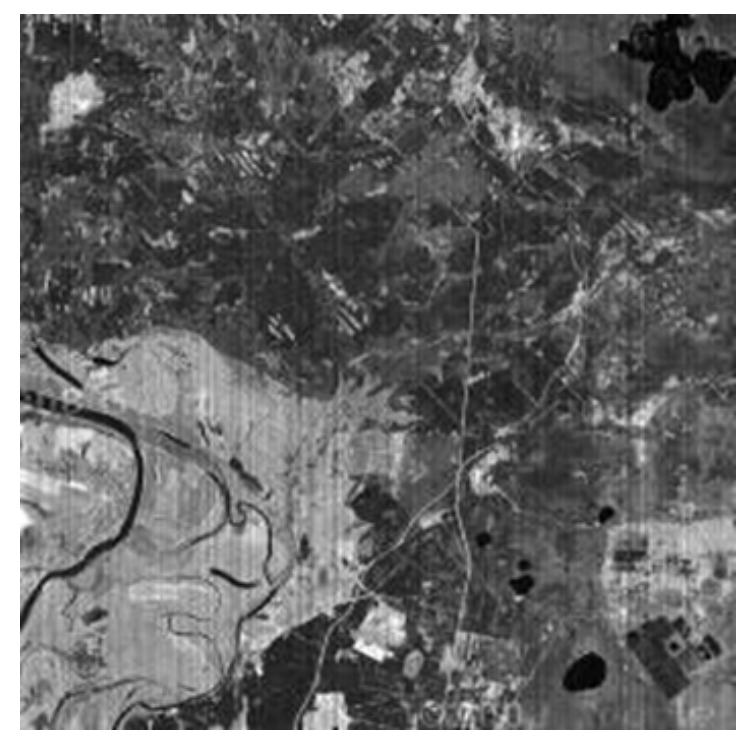

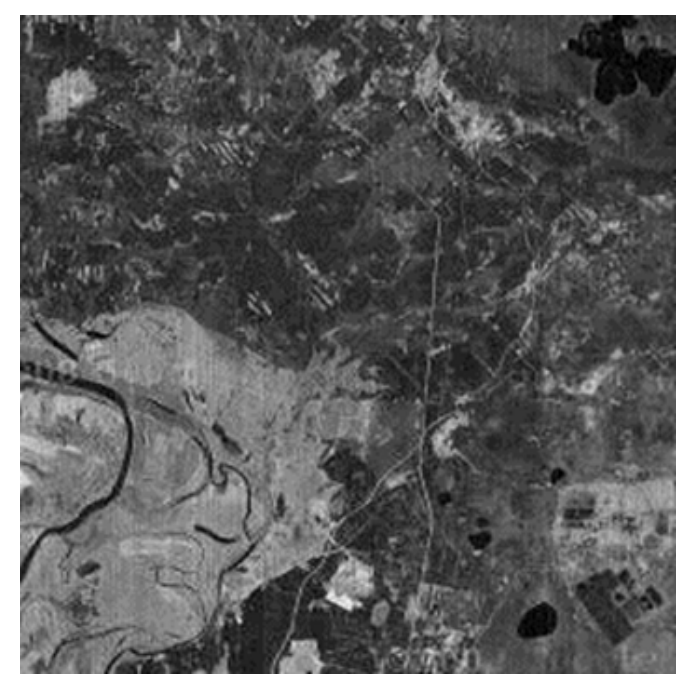

Figure 1. Example of image filtration with multiplicative synchronous noise: (a) is the initial image $\mathrm{MSD}=7.4184$; (b) is the image transferred $\mathrm{MSD}=2.77$

Fig. 1 shows the result of using Theorems 1 and 5 for filtration of images with synchronous multiplicative noises [10]. Filtration is performed through diagonal filtration in a quasi-two-dimensional spectral Walsh space. Image recovery follows this formula:

$$
B=\mathbf{H}_{\mathrm{w}} \mathbf{H}_{\mathrm{uu}} \mathbf{C}
$$

where $\mathbf{B}$ is the matrix of the recovered image; $\mathbf{C}$ is the matrix of spectral coefficients; $\mathbf{H}_{w}$ is a WalshHadamard matrix; $\mathbf{H}_{\mathbf{u u}}$ is a matrix whose rows are made up of coefficients of row $h_{u u}$ diagonal filters:

$$
h_{u u}=\frac{\left(\sum_{\mathrm{G}}\right)_{u u}-\left(\sum_{\eta}\right)_{u u}}{\left(\sum_{G}\right)_{u u}}
$$

where $\left(\sum_{\mathrm{G}}\right)_{u u}$ and $\left(\sum_{\eta}\right)_{u u}$ are co-variation matrices of image rows with noises and of the noise itself, correspondingly, computed in a spectral space.

\section{Conclusion}

Removal of distortions that emerge during formation and transfer of aerospace images is the principal task of preliminary processing of compression algorithm image compression, to form prerequisites for using the images.

Large volumes of data to process make us constantly look for methods and tools of acceleration, and improve the mathematical toolset for processing them as discrete transformations at their finite intervals. Introducing the notion of finiteness, we can avoid the contradictions that arise from the Fourier transformation, and conduct spatial-spectral analysis of images that are in a general way described by a non-stationary arbitrary process. In this respect, using the principal statements and conclusions of the theory of discrete signal at finite intervals is quite justified. This theory is based on choosing the most acceptable option of constructing a basic function system from the whole range of VCFs, for which signal shift is defined as bitwise addition of numbers in a certain module. Using this method considerably reduces computing expenditure (tenfold or more), while the quality of preliminary processing of 
aerospace images considerably increases. Examples of using the described approaches in development of systems that are to implement practical tasks are given in [15].

\section{References}

1. Gonzalez R.C., Woods R.E. Digital Image Processing: Prentice Hall. New Jersey. 2002. P. 1072.

2. Kostrov B.V. The Features of Aerospace Image Forming by Radar Facilities // Proektirovaniye i tekhnologiya elektronnykh sredstv. 2011. Vol. 1. Pp. 41-43.

3. Kostrov B.V. Correlation-extremum method of digital signal detection // Tsifrovaya obrabotka signalov. 2011. № 2. Pp. 46-50.

4. Zlobin V.K., Kolesenkov A.N., Kostrov B.V., Correlation-extremum methods of aerospace image alignment // Vestnik Ryazanskogo gosudarstvennogo radiotekhnicheskogo universiteta. 2011. № 37. Pp. 12-17.

5. Zlobin V.K., Kostrov B.V., Sablina V.A. Place and role of sequential analysis methods in processing of aerospace images // Radiotekhnika. 2012. № 3. Pp. 64-72.

6. Kolesenkov A.N., Kostrov B.V. Method of thinning of basic functions in correlation-extremum algorithms // Voprosy radioelektroniki. - 2010. Issue 1. - Series OT. Pp. 176 - 184.

7. Zlobin V.K., Kostrov B.V., Sablina V.A. Algorithm of sequential filtration of group noises in images // Vestnik Ryazanskogo gosudarstvennogo radiotekhnicheskogo universiteta. 2009. № 30. Pp. 37.

8. Kostrov B.V., Sablina V.A. Adaptive filtration of images with structural distortions // Tsifrovaya obrabotka signalov. 2008. № 4. Pp. 49-53.
9. Kostrov B.V., Upakova A.G. Quasi-twodimensional filtration of synchronous noises in images // Proektirovaniye i tekhnologiya elektronnykh sredstv. 2012. № 1. Pp. 32-35.

10. Kostrov B.V., Grinchenko N.N., Stepanov D.S., Upakova A.G. Algorithm of image transfer with recovery of the constant component // Izvestya Tulskogo gosudarstvennogo universiteta. Tekhnicheskiye nauki. 2013. № 9-1. Pp. 244-249.

11. Ahmed N., Rao K. R. Orthogonal Transforms for Digital Signal Processing.: Springer-Verlag Berlin, Heidelberg New York. 1975. P. 250.

12. Kostrov B.V., Kostrov V.V. Method of fold computation in a non-trigonometrical finite basis // Radiotekhnicheskiye i telekommunikatsionnye systemy. 2015. № 2 (18). Pp. 66-73.

13. Kolesenkov A., Kostrov B., Ruchkina E., Ruchkin V. Anthropogenic situation express monitoring on the base of the fuzzy neural networks // Proceedings 2014 3rd Mediterranean Conference on Embedded Computing, MECO 2014 - Including ECyPS 20143. 2014. Pp. 166-168.

14. Ruchkin V.N., Kostrov B.V., Kolesenkov A.N., Ruchkina E.V. Algorithms of Fire seat Detection, Modeling Their Dynamics and Observation of Forest Fires via Communication Technologies // Proceedings - 2015 4th Mediterranean Conference on Embedded Computing. BUDVA. 2015. Pp. 254 257.

15. Kolesenkov A.N., Kostrov B.V., Ruchkin V.N. Emergencies monitoring and preventing // Proceedings - 2013 2nd Mediterranean Conference on Embedded Computing, MECO - 2013. 2013. Pp. 263-265. 